\title{
Spontaneous Remission of a Non-small Cell Lung Cancer Possibly Caused by Anti-NY-ESO-1 Immunity
}

Yoichi Nakamuraa , M.D., Yuji Noguchi', M.D., Eiichi Satoh ${ }^{c}$, M.D., Akiko Uenaka ${ }^{d}$, Ph.D., Shuichiro Sato ${ }^{d}$, M.D., Takeshi Kitazaki ${ }^{e}$, M.D., Tetsuro Kanda ${ }^{\mathrm{e}}$, M.D., Hiroshi Soda ${ }^{a}$, M.D., Eiichi Nakayama ${ }^{d}$, M.D., and Shigeru Kohno ${ }^{a}$, M.D.

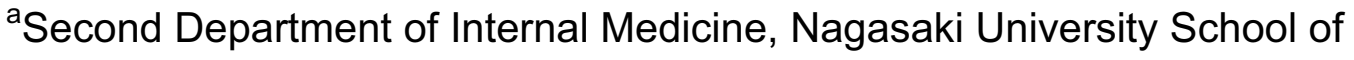
Medicine, Sakamoto 1-7-1, Nagasaki, Japan; ${ }^{b}$ Department of Internal Medicine, Naka-Tsushima Hospital, Tsushima, Japan; 'Department of Pathology, Tokyo

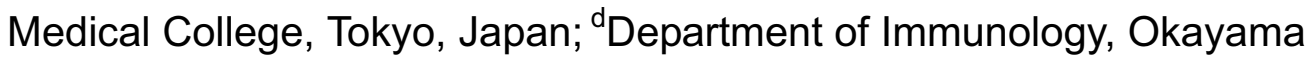
University Graduate School of Medicine and Dentistry, Okayama, Japan; eDepartment of Internal Medicine, Goto Central Hospital, Goto, Japan.

Correspondance: Second Department of Internal Medicine, Nagasaki University School of Medicine, 1-7-1 Sakamoto, Nagasaki 852-8501, Japan Phone: +81-(95)-849-7274; Fax: +81-(95)-849-7285

E-mail: yi-nakamu@umin.ac.jp 


\section{Abstract}

Spontaneous remission of malignant tumors is rare and the biological

mechanism of such remission has not been addressed. We report the case of a 71-year-old Japanese patient with non-small cell lung cancer with a right hilar tumor and pleural dissemination that spontaneously regressed. NY-ESO-1 is a cancer/testis antigen that can elicit specific immune responses in patients with cancer. Strong anti-NY-ESO-1 immunity was detected in this patient. His tumor cells expressed NY-ESO-1 and MHC class I molecules. Anti-NY-ESO-1 immunity might have contributed to spontaneous remission in this patient. 


\section{Introduction}

Spontaneous remission is extremely rare in patients with non-small cell lung cancer (NSCLC). Any underlying mechanism of this remission remains unclear [1]. A systemic reaction, such as an immune response to tumors, seemed to be a possible causative mechanism. However, there have been no reports indicating immunoreaction-mediated spontaneous remission in patients with NSCLC.

NY-ESO-1 was originally identified in esophageal cancer by serological expression cloning using autologous patient serum and found to be a cancer/testis antigen that is expressed in cancer and testis, but not in normal adult somatic tissues [2, 3]. This antigen has made one of the fastest transitions from molecular, cellular, and immunological descriptions to a vaccine and immunotherapy candidate. NY-ESO-1 has already been tested in various formulations in more than 30 clinical trials worldwide, and its main characteristic resides in its capacity to elicit spontaneous antibody and T-cell responses in a proportion of patients with cancer [4]. Here, we present the case of a patient with NSCLC that spontaneously regressed, possibly mediated by anti-NY-ESO-1 immunity. 


\section{Case report}

A 71-year-old man was referred to Goto Central Hospital, Nagasaki, Japan in November 2004 for further examination of abnormal shadows on his chest x-ray. A chest computed tomography $(\mathrm{CT})$ revealed a right hilar tumor, measuring $3 \times 3$ $\mathrm{cm}$, and right multiple focal pleural thickenings (Fig. 1A). The patient underwent a thoracoscopy and tumor specimens were collected from the right pleural thickening. The pathological diagnosis was poorly differentiated adenocarcinoma with positive staining for cytokeratin 7 and negative staining for cytokeratin 20 (Fig. 1B). The clinical diagnosis was c-T4NOM0 stage IIIB NSCLC. The patient refused to receive any treatment at that time. A follow-up chest CT showed the disappearance of pleural dissemination and shrinkage of the right hilar tumor (Fig. 1A).

\section{Materials and Methods}

Blood was drawn from the patient with informed consent. Collected serum samples were frozen until use. Peripheral blood mononuclear cells (PBMC) were isolated by density gradient centrifugation. CD4 and CD8 T-cells were obtained from PBMC using CD4 and CD8 microbeads, respectively, with columns and 
magnetic devices (Miltenyi Biotec, Auburn, CA). Residual cells were used as CD4- and CD8-depleted cells. These cells were stored in liquid $\mathrm{N}_{2}$ until use. Antibody responses to cancer/testis antigens were evaluated by enzyme-linked immunosorbent assay (ELISA) as described elsewhere [5]. NY-ESO-1 is composed of 180 amino acids [2]. Twenty-eight 18-mer NY-ESO-1 overlapping peptides, spanning 1-173 amino acids of N-terminal NY-ESO-1, and one 30-mer C-terminal peptide, spanning 151-180 amino acids, were synthesized with standard solid-phase methods using a Multiple Peptide Synthesizer (AMS422; ABIMED, Langenfeld, Germany) [6]. To detect T-cell response to NY-ESO-1, CD4 and CD8 T-cells $\left(2 \times 10^{6}\right)$ were cultured with irradiated (30 Gy) CD4- and CD8-depleted cells $\left(2 \times 10^{6}\right)$ in the presence of 28 18-mer NY-ESO-1 overlapping peptides and a 30 -mer C-terminal peptide $(1 \mu \mathrm{g} / \mathrm{ml}$ for each peptide) in AIM-V (Invitrogen, Carlsbad, CA) with 5\% heat-inactivated pooled human serum with 10 units/ml IL-2 (Takeda Chemical Industry, Osaka, Japan) and 10 $\mathrm{ng} / \mathrm{ml} \mathrm{IL-7} \mathrm{(Peprotech,} \mathrm{London,} \mathrm{UK)} \mathrm{in} \mathrm{a} 24$-well plate at $37^{\circ} \mathrm{C}$ in a $5 \% \mathrm{CO}_{2}$ atmosphere for 12 days. IFN $\gamma$ secretion assays with $2 \times 10^{5}$ cells were performed according to manufacturer's protocol [6]. Immunohistochemistry was performed as described elsewhere [7]. 


\section{Results}

Antibody responses to cancer/testis antigens were determined by enzyme-linked immunosorbent assay using $1 \mu \mathrm{g}$ of NY-ESO-1 (०), SSX-2 (•), SSX-4 ( $\mathbf{\Delta})$, and

XAGE-1 ( $\mathbf{~})$ recombinant proteins because of their high expression in lung cancer and strong immunogenicity in patients with NSCLC. A high titer of IgG antibody specific to NY-ESO-1 was detected and observed throughout the period starting July 2006 (Fig. 2A). We also observed strong CD4 and CD8 T-cell responses specific to NY-ESO-1 in an assay for interferon gamma (IFN $\gamma$ ) secretion. Thus, integrated anti-NY-ESO-1 immunity consisting of antibody with CD4 and CD8 T-cell responses was detected in the patient (Fig. 2B). Immunohistochemical staining was performed for NY-ESO-1, MHC class I, and $\mathrm{CD}^{+}$T-cells. Cytoplasmic expression of NY-ESO-1 was observed in $50-60 \%$ cancer cells (Fig. 3A). MHC class I was stained on the cell surface of $30-40 \%$ cancer cells (black arrows) (Fig. 3B). $\mathrm{CD}^{+}$T-cells were observed in the interface between the stromal and tumor tissues (black arrows) and also within the tumor tissue (white arrows) (Fig. 3C). $\mathrm{CD}^{+}$T-cells in tumor tissue were counted using a 40x objective lens in 10 fields and more than 30 cells were observed in each 
field. In addition, double staining of CD25 (brown) and FOXP3 (red) showed that $\mathrm{CD}^{+} 5^{+} \mathrm{FOXP}^{+}$T-cells (black arrows) were mainly distributed in stromal tissue and that $\mathrm{CD}^{2} 5^{+} \mathrm{FOXP3}^{-}$T-cells (white arrows) were observed in tumor tissue in this patient (Fig. 3D).

As shown in Figure 1A, a follow-up chest CT scan in February 2006 showed the disappearance of pleural dissemination, while the right hilar tumor had increased to $4.5 \times 3 \mathrm{~cm}$. Once the patient agreed to receive treatment, radiation (a total of 60 Gy in 30 fractions) against the tumor was started in March, 2006; this was effective and resulted in a partial response [5]. As of September 2007, the patient was doing well and no recurrence of pleural dissemination had been observed.

\section{Discussion}

We detected a high titer of IgG antibody specific to NY-ESO-1 from a patient with NSCLC that spontaneously regressed. We also observed anti-NY-ESO-1 CD4 and CD8 T-cell responses from his lymphocytes. Integrated anti-NY-ESO-1 immunity was elicited by tumor cells expressing NY-ESO-1. Immunohistochemical staining of tumor-infiltrating lymphocytes (TIL) showed a 
high number of CD8 TIL at the tumor sites. Although it was not verified that CD8 TILs were NY-ESO-1-specific T cells, systemic NY-ESO-1 immunity evoked in this patient might have contributed to tumor regression.

The mechanism of spontaneous remission remains unclear. However , it was suggested that systemic immunity against NY-ESO-1 contributed to the tumor regression in this case. More analysis regarding tumor microenvironment could provide the exact mechanism of tumor remission in NSCLC.

No authors have any potential conflicts of interest regarding this manuscript. 


\section{References}

[1] Kappauf H, Gallmeier WM, Wünsch PH, Mittelmeier HO, Birkmann J, Büschel G, et al. Complete spontaneous remission in a patient with metastatic non-small-cell lung cancer. Ann Oncol 1997;8:1031-9.

[2] Chen YT, Scanlan MJ, Sahin U, Türeci O, Gure AO, Tsang S, et al. A testicular antigen aberrantly expressed in human cancers detected by autologous screening. Proc Natl Acad Sci USA 1997;94:1914-8.

[3] Jager E, Chen YT, Drijfhout JW, Karbach J, Ringhoffer M, Jäger D, et al. Simultaneous humoral and cellular immune response against cancer-testis antigen NY-ESO-1: definition of human histocompatibility leukocyte antigen (HLA)-A2-binding peptide epitopes. J Exp Med 1998;187:265-70.

[4] Gnjatic S, Nishikawa H, Jungbluth AA, Güre AO, Ritter G, Jäger E, et al. NY-ESO-1: review of an immunogenic tumor antigen. Adv Cancer Res 2006;95:1-30.

[5] Kawabata R, Wada H, Isobe M, Saika T, Sato S, Uenaka A, et al. Antibody response against NY-ESO-1 in CHP-NY-ESO-1 vaccinated patients. Int J Cancer 2007;120:2178-84.

[6] Uenaka A, Wada H, Isobe M, Saika T, Tsuji K, Sato E, et al. T cell 
immunomonitoring and tumor responses in patients immunized with a complex of cholesterol-bearing hydrophobized pullulan (CHP) and NY-ESO-1 protein. Cancer Immun 2007;7:9.

[7] Sato E, Olson SH, Ahn J, Bundy B, Nishikawa H, Qian F, et al. Intraepithelial $\mathrm{CD}^{+}$tumor-infiltrating lymphocytes and a high $\mathrm{CD} 8^{+} /$regulatory $\mathrm{T}$ cell ratio are associated with favorable prognosis in ovarian cancer. Proc Natl Acad Sci USA 2005;102:18538-43.

[8] Therasse P, Arbuck SG, Eisenhauer EA, Wanders J, Kaplan RS, Rubinstein L, et al. New guidelines to evaluate the response to treatment in solid tumors. $J$ Natl Cancer Inst 2000;92:205-16. 


\section{Figure Legends}

Fig. 1. (A) Chest computed tomography. White arrows indicate the right hilar tumor and black arrows indicate pleural disseminations. (B) Staining of a biopsy sample of pleural metastasis. The pathological diagnosis was poorly differentiated adenocarcinoma with positive staining for cytokeratin 7 and negative staining for cytokeratin 20 .

Fig. 2. Immune response to NY-ESO-1 in the patient. (A) Enzyme-linked immunosorbent assay (ELISA). Antibody response to cancer/testis antigens was

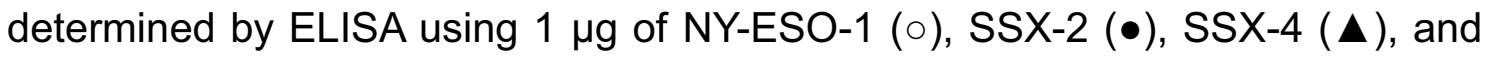
XAGE-1 (-) recombinant proteins. (B) Interferon gamma (IFNY) secretion assays. CD4 and CD8 T-cells $\left(2 \times 10^{6}\right)$ purified from peripheral blood mononuclear cells (PBMC) using magnetic cell sorting were cultured for 12 days with NY-ESO-1 peptides. The cells $\left(2 \times 10^{5}\right)$ were assayed for IFNy secretion in response to PFA-treated autologous CD4- and CD8-depleted PBMC $\left(2 \times 10^{5}\right)$ pre-pulsed or unpulsed with NY-ESO-1 peptides using fluorescence-activated cell sorting. Values higher than $0.1 \%$ were considered to be significant. 
Fig. 3. Immunohistochemistry of a biopsy sample from pleural metastasis. (A) NY-ESO-1. Cytoplasmic staining was observed. (B) MHC class I. Surface staining was observed as indicated by black arrows. (C) $C D 8^{+} \mathrm{T}$-cells were observed in the interface between stromal and tumor tissues (black arrows) and also within the tumor tissue (white arrows). (D) Double staining of CD25 and FOXP3. Regulatory T-cells (black arrows) were detected by double staining of CD25 (brown, cell surface staining) and FOXP3 (red, nuclear staining). White arrows indicate $\mathrm{CD} 25^{+} \mathrm{FOXP3}^{-} \mathrm{T}$-cells. A bold line indicates the border between the stroma and the tumor. 
(A)
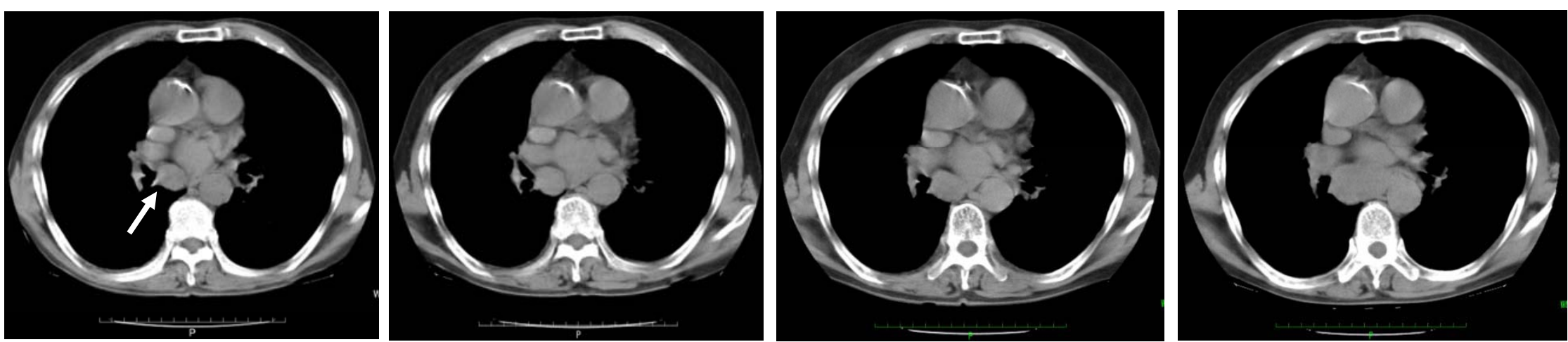

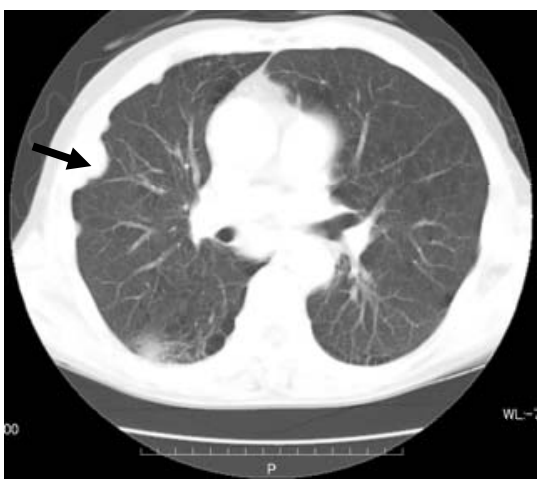

November, 2004

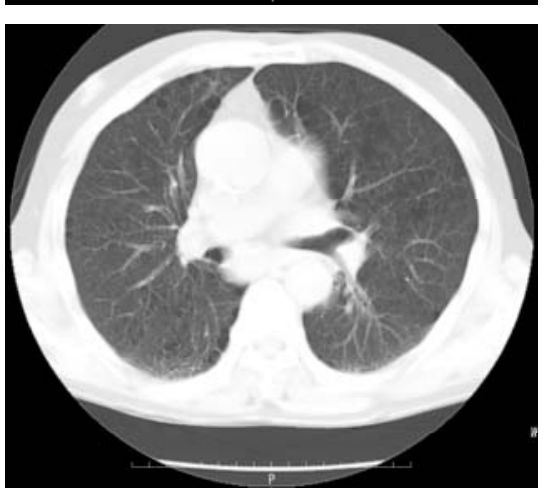

April, 2005

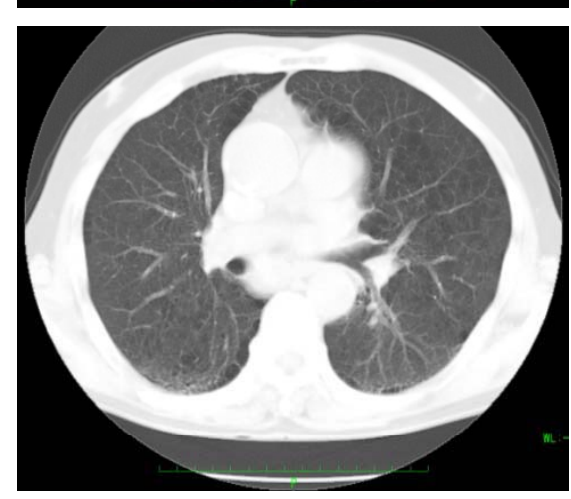

October, 2005

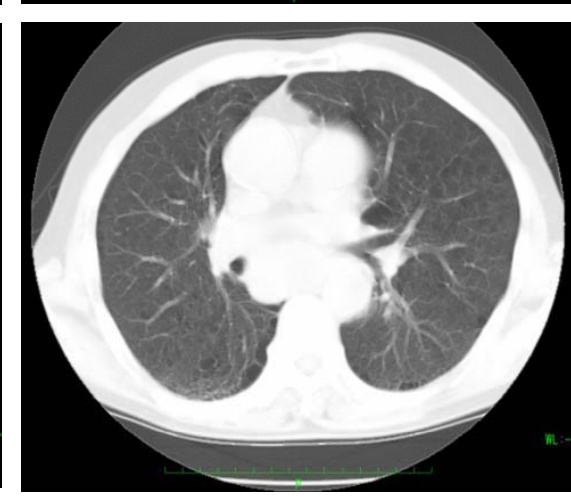

February, 2006

(B)

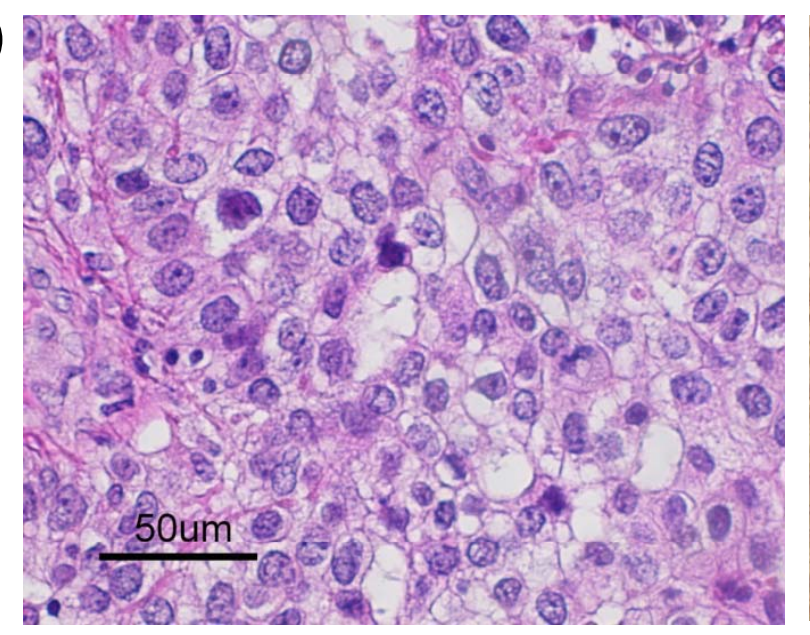
HE

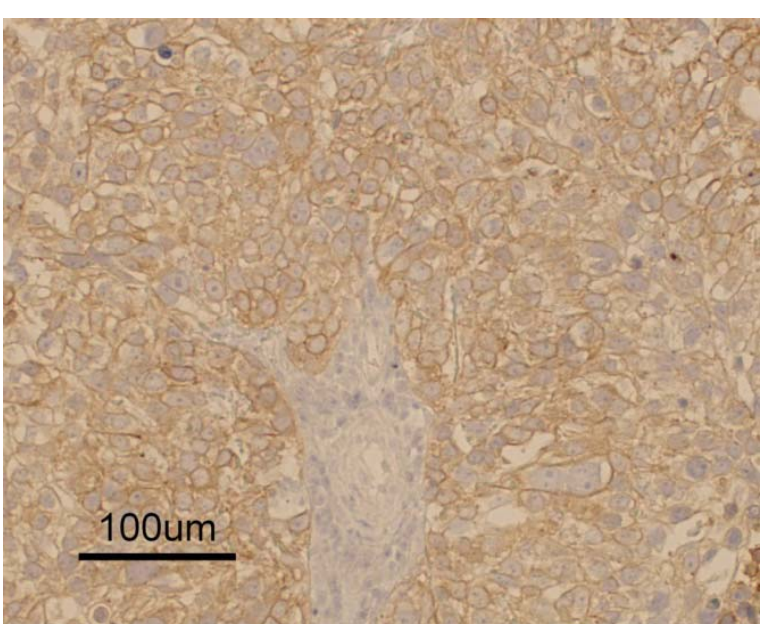

Cytokeratin 7 100um

Cytokeratin 20 
(A)

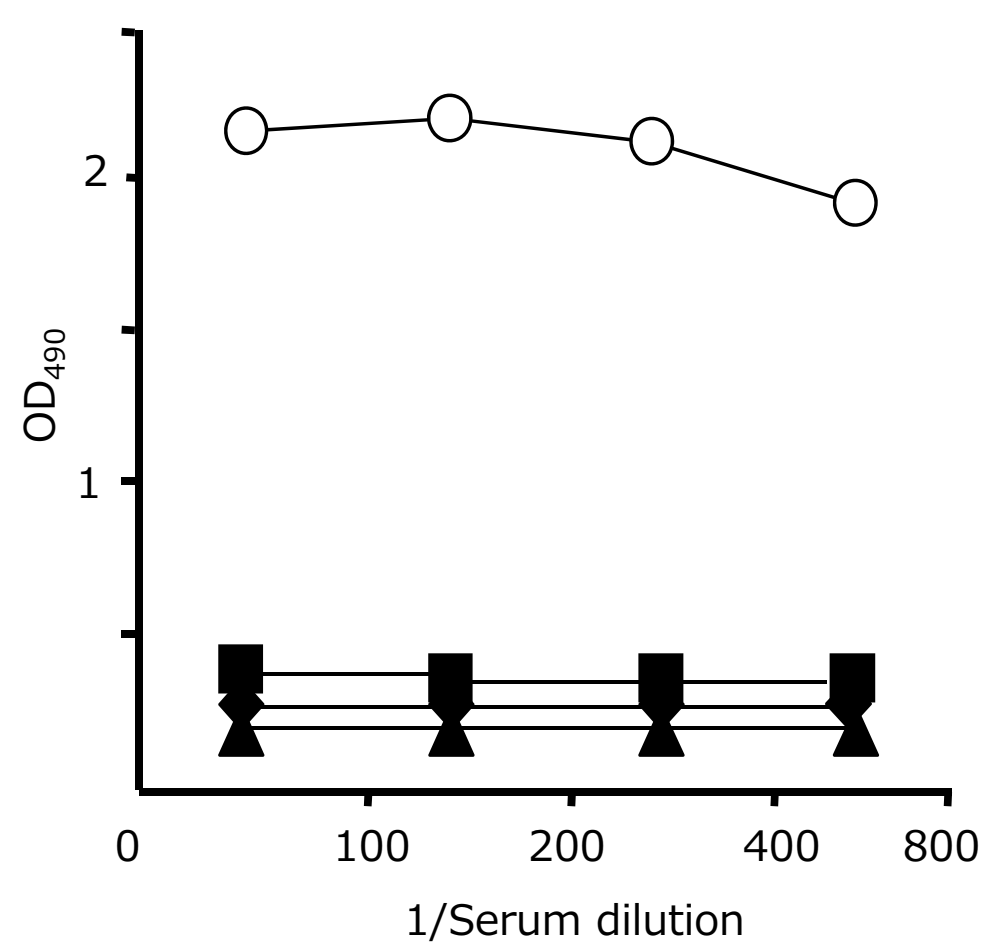

(B)
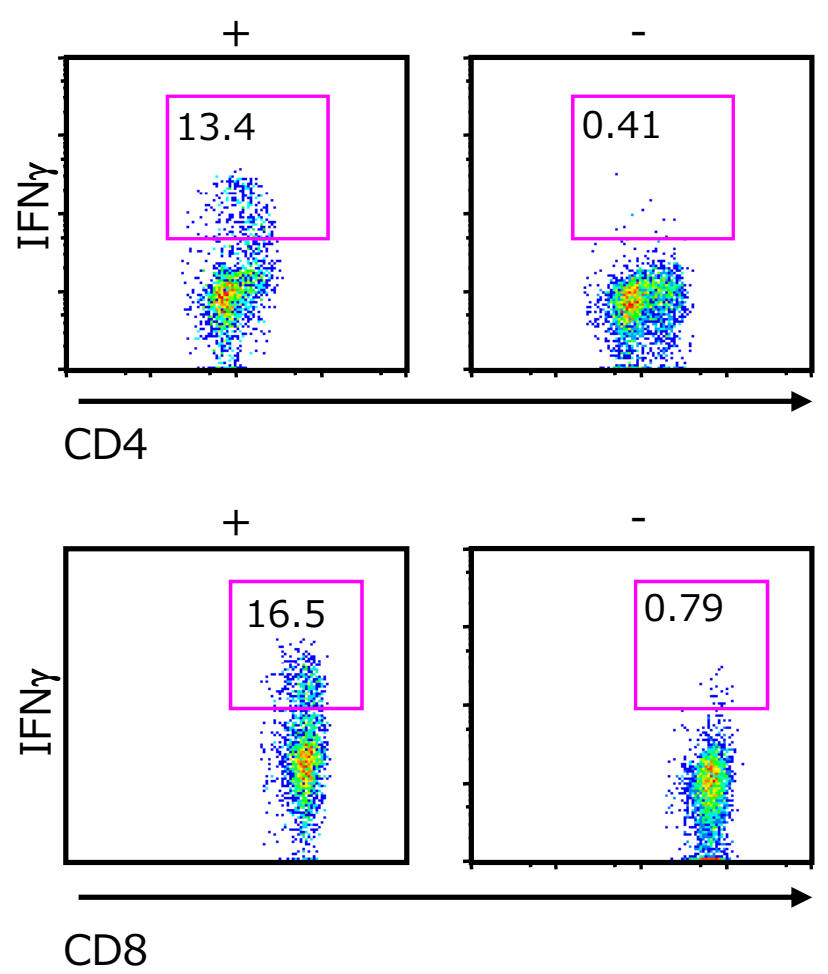
(A) NY-ESO-1

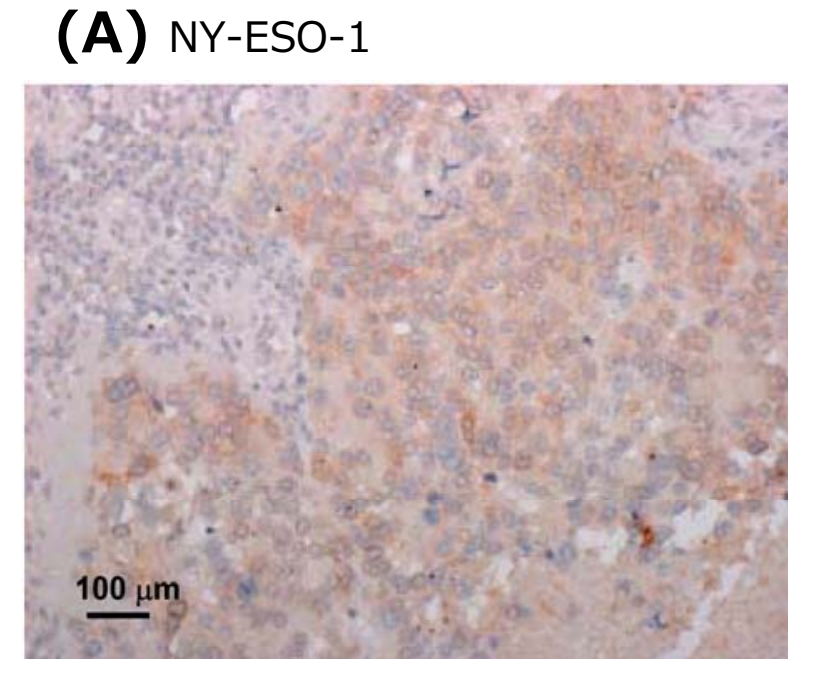

(C) $\mathrm{CD} 8$

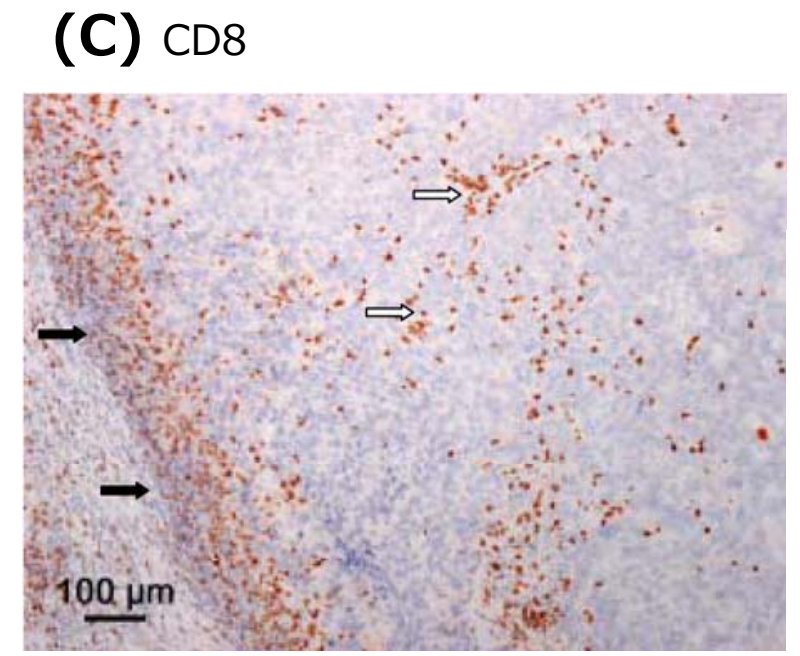

(B) MHC class I

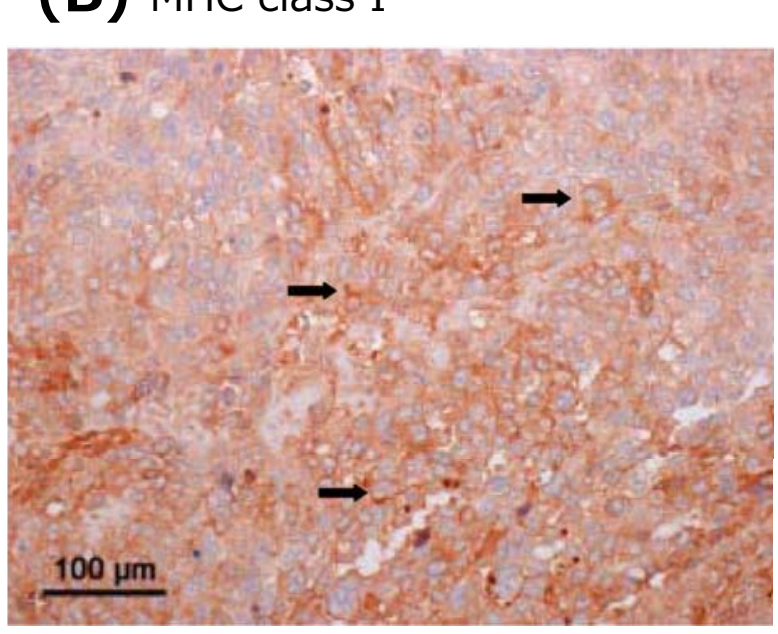

(D) $\mathrm{CD} 25 / \mathrm{FOXP3}$

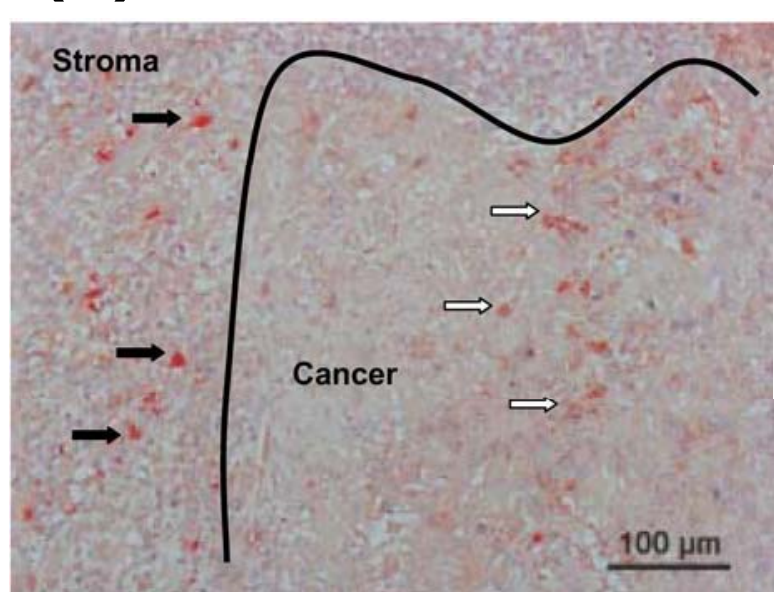$$
\text { . }
$$

.

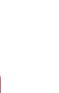

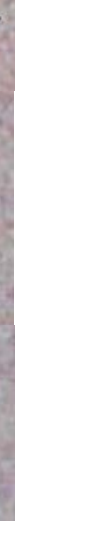

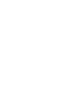

